\title{
2016 update to The American Association for Thoracic Surgery consensus guidelines: Ischemic mitral valve regurgitation
}

\author{
The American \\ Association For \\ Thoracic Surgery \\ Ischemic Mitral \\ Regurgitation \\ Consensus \\ Guidelines Writing \\ Committee: \\ Irving L. Kron, MD, ${ }^{a}$ Damien J. LaPar, MD, MSc, \\ Michael A. Acker, MD, ${ }^{b}$ David H. Adams, MD, \\ Gorav Ailawadi, MD, ${ }^{\mathrm{a}}$ Steven F. Bolling, $\mathrm{MD},{ }^{\mathrm{d}}$ \\ Judy W. Hung, MD, ${ }^{e}$ D. Scott Lim, MD, ${ }^{f}$ \\ Michael J. Mack, MD, ${ }^{\mathrm{g}}$ Patrick T. O'Gara, MD, \\ Michael K. Parides, PhD, ${ }^{\mathrm{i}}$ and John D. Puskas, MD
}

We are very pleased to update The American Association for Thoracic Surgery (AATS) Consensus Guidelines on ischemic mitral valve regurgitation (IMR) (Figure 1). These Guidelines were developed based on the results of published randomized clinical trials, large observational studies, and the expert opinion of the authors. Subsequent to the publication of the 2015 AATS IMR Guidelines, ${ }^{1}$ the 2-year follow-up results of the Cardiothoracic Surgical Trials Network (CTSN) severe and moderate ischemic mitral regurgitation (MR) trials were published. ${ }^{2,3}$ The 2year data from the Severe MR (SMR) trial demonstrated that nearly half of alive mitral repair patients developed recurrent MR with a low percentage developing severe MR. However, there were significantly more episodes of heart failure and cardiovascular-related hospitalizations in the repair group, so there certainly appears to be a concordance between these echocardiographic and clinical results. Consistent with these findings, we have modified the language and levels of evidence (LOE) for the Writing Committee's guidelines for severe IMR (Table 1).

The specific changes to the Writing Committee's guidelines for severe IMR concern the fundamental change in

\footnotetext{
From the aDivision of Thoracic and Cardiovascular Surgery, Department of Surgery, and ${ }^{\mathrm{f}}$ Division of Pediatric Cardiology, Departments of Pediatrics and Medicine, University of Virginia, Charlottesville, Va; 'bivision of Cardiovascular Surgery, Department of Surgery, University of Pennsylvania School of Medicine, Philadelphia, Pa; ${ }^{\mathrm{c} D e p a r t m e n t}$ of Cardiac Surgery, Mount Sinai Medical Center; ${ }^{\mathrm{d}}$ Department of Cardiac Surgery, University of Michigan, Ann Arbor, Mich; ${ }^{\mathrm{e} D i v i s i o n}$ of Cardiology, Department of Medicine, Massachusetts General Hospital; ${ }^{\mathrm{g}}$ Department of Cardiovascular Surgery, Heart Hospital Baylor Plano, Baylor Health Care System, Plano, Tex; ${ }^{\mathrm{h} C a r d i o v a s c u l a r}$ Division, Department of Medicine, Brigham and Women's Hospital, Boston, Mass; and ${ }^{\mathrm{i}}$ The International Center for Health Outcomes and Innovation Research, Department of Population Health Science and Policy, Icahn School of Medicine at Mount Sinai, New York, NY.

Received for publication May 31, 2016; revisions received Nov 15, 2016; accepted for publication Nov 16, 2016; available ahead of print Feb 9, 2017.

Address for reprints: Irving L. Kron, MD, Division of Thoracic and Cardiovascular Surgery, Department of Surgery, University of Virginia School of Medicine, PO Box 800679, Charlottesville, VA 22908 (E-mail: ilk@virginia.edu).

J Thorac Cardiovasc Surg 2017;153:1076-9

0022-5223/\$36.00

Copyright (c) 2017 by The American Association for Thoracic Surgery

http://dx.doi.org/10.1016/j.jtcvs.2016.11.068
}

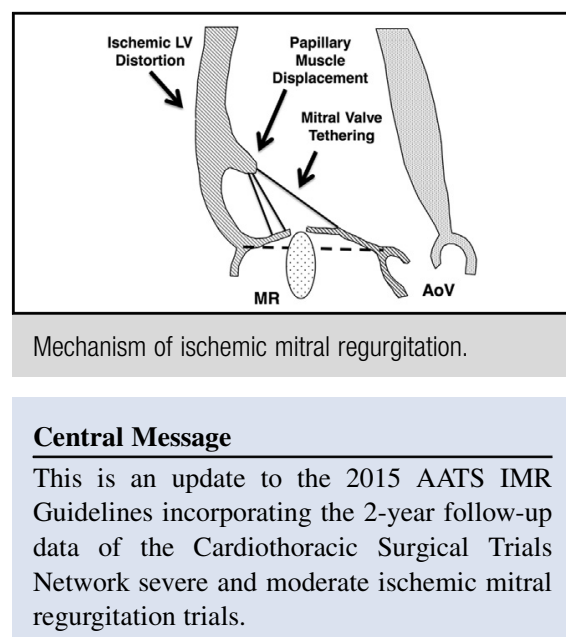

See Editorial Commentary page 1080.

the level of evidence guiding the recommendation for $\mathrm{LOE}$ A to LOE B and a change in the language of the recommendation to make it more consistent with that of an LOE B guideline. The rationale for changing the LOE for these recommendations were primarily driven by our belief that, after reviewing the best-available evidence, several of the randomized trials and prospective series for the surgical treatment of IMR currently available are simply not large enough to support LOE A classification. In addition, we have added to each Guideline that surgical correction of IMR "is reasonable" and "may be considered" in patients "who remain symptomatic despite Guideline-directed medical and cardiac device therapy." The recommendations for performance of mitral valve replacement in the setting of basal aneurysm/ dyskinesia are based on results from the CTSN SMR trial, which demonstrated that the presence of basal aneurysms is associated with recurrent MR following mitral repair. ${ }^{4}$

The original Guidelines for SMR are as follows:

- In the presence of basal aneurysm/dyskinesis, significant echocardiographic evidence of leaflet tethering, or moderate to severe left ventricle remodeling

Scanning this QR code will take you to the full updated version of the guideline.

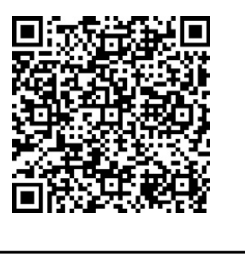




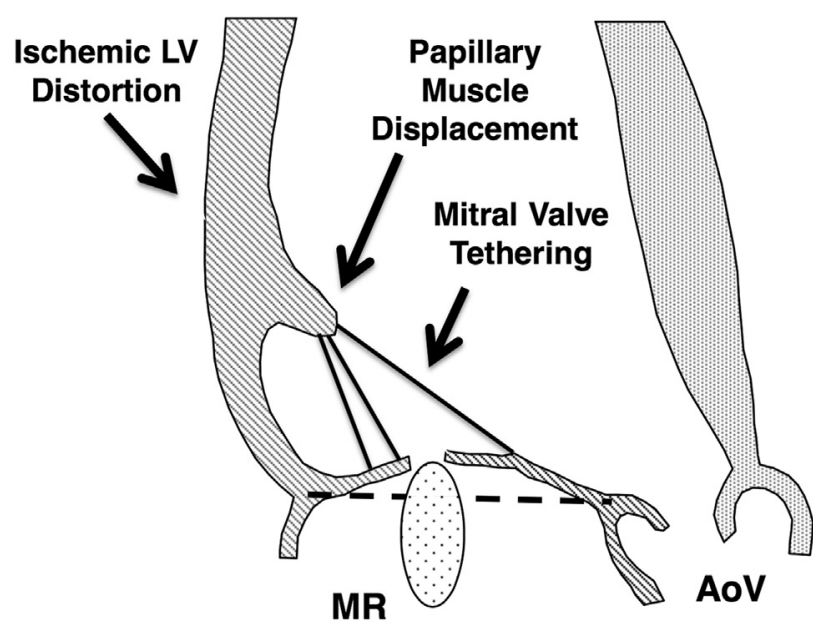

FIGURE 1. Diagram of pathophysiologic mechanism of ischemic mitral regurgitation. $L V$, Left ventricle; $M R$, mitral regurgitation; $A o V$, aortic valve.

(left ventricular end diastolic diameter [LVEDD] $>65$ $\mathrm{mm}$ ), patients should consider mitral valve replacement (class of recommendation [COR] IIa, LOE A).

- In the absence of basal aneurysm/dyskinesis, echocardiographic evidence of significant leaflet tethering, or moderate to severe left ventricle remodeling (LVEDD), patients should consider mitral valve repair with an undersized, complete rigid ring (COR IIb, LOE B).

The updated Guidelines for SMR are as follows:

- Mitral valve replacement is reasonable in patients with severe IMR who remain symptomatic despite Guidelinedirected medial and cardiac device therapy, and who have a basal aneurysm/dyskinesis, significant leaflet tethering, and/or severe left ventricle dilation (LVEDD $>6.5 \mathrm{~cm})$ (COR IIa, LOE B).

- Mitral valve repair with an undersized complete rigid annuloplasty ring may be considered in patients with severe IMR who remain symptomatic despite Guidelinedirected medical and cardiac device therapy and who do not have a basal aneurysm/dyskinesis, significant leaflet tethering, or severe left ventricle enlargement (COR IIb, LOE B).

The 2-year results from the CTSN Moderate MR trial have principally changed the opinion of the Writing Committee and subsequent Guidelines. We were concerned initially that the presence of moderate MR in patients who undergo mitral valve repair would lead to further significant MR and clinical sequelae. However, at 2-year follow-up this did not occur. Overall, patients seemed to do just as well with coronary artery bypass grafting (CABG) alone compared with combined $\mathrm{CABG}$ with mitral valve repair with the exception of improvement in exercise capacity in the repair group. The mitral valve repair group perioperatively had a higher neurologic event rate and increased arrhythmias. Therefore, there was a cost to adding mitral valve repair to these patients.
We have changed the Guidelines specifically related to performance of mitral valve repair for patients with moderate MR. The updated Guidelines (Table 1) now recommend that in patients with moderate IMR undergoing CABG, mitral valve repair with an undersized complete angioplasty ring "may be considered." This differs from the initial Guideline, which stated that patients with moderate MR "should undergo" concomitant mitral valve repair and identified certain clinical situations where concomitant mitral valve repair may be appropriate. As a result, the Writing Committee emphasizes the importance of individual surgeon experience and clinical expertise to determine when concomitant mitral repair is indicated for the surgical treatment of moderate MR. It seems that in the majority of situations, CABG alone has equivalent results. Furthermore, we updated the LOE supporting these Guidelines from LOE A to a more appropriate LOE B to be consistent with the bestavailable supporting evidence for these recommendations.

The original Guideline for moderate MR was as follows:

- Patients with moderate IMR undergoing CABG should undergo concomitant mitral valve repair with an undersized, complete rigid annuloplasty ring to mitigate recurrence of MR in patients who have heart failure symptoms; those with significant mitral annular dilation; and those in whom bypassable, hibernating, viable myocardium supporting the papillary muscle(s) is thought to be minimal (COR IIb, LOE A).

The updated Guideline for moderate MR is as follows:

- In patients with moderate IMR undergoing CABG, mitral valve repair with an undersized complete rigid annuloplasty ring may be considered (COR IIb, LOE B).

The Guidelines related to the performance of either mitral valve replacement versus repair for IMR did not change in this update. The LOE supporting these Guidelines also did not change in this update. As reviewed in our original Guidelines, in an important clinical trial comparing patients treated with mitral valve repair for IMR, Spoor and colleagues $^{5}$ demonstrated a significant benefit of use of small, complete rigid annuloplasty rings compared with flexible rings (5-fold greater incidence of recurrent MR with flexible rings). These findings were supported in a subsequent multivariate analysis performed by Silberman and colleagues, ${ }^{6}$ which identified that the type of annuloplasty ring (rigid vs flexible) was an important predictor of residual and recurrent MR after mitral repair for IMR. For the performance of mitral valve replacement, results from a seminal clinical trial reported by Yun and colleagues ${ }^{7}$ demonstrated the superiority of a complete chordalsparing mitral valve replacement (compared with partial chordal-sparing replacement) with improved preservation of left ventricle volume and function. Thus, the Writing Committee continues to support the performance of a 
TABLE 1. Summary comparison of existing societal guidelines and updated The American Association for Thoracic Surgery Guidelines for Surgical Treatment of Ischemic Mitral Regurgitation

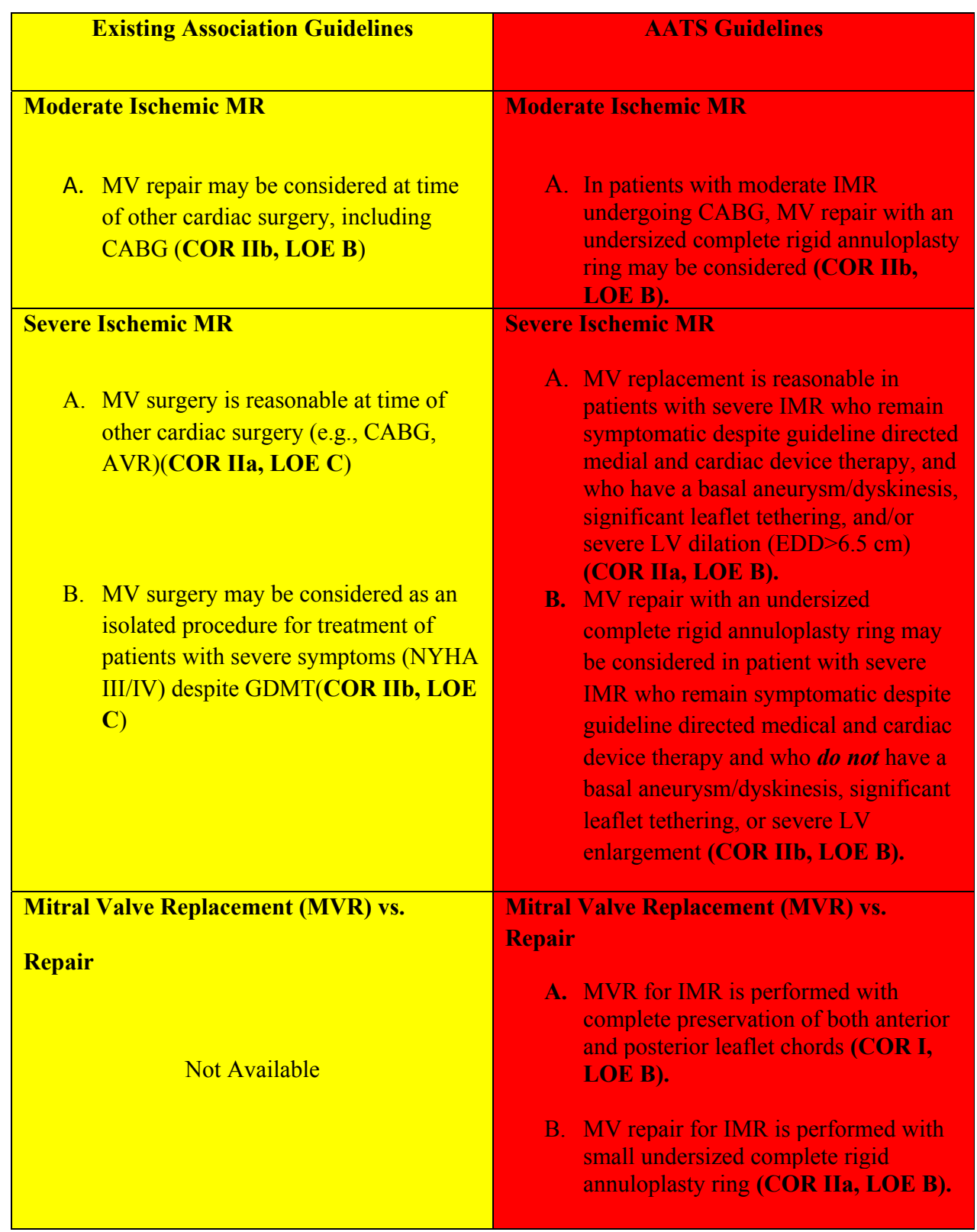

$\overline{A A T S}$, The American Association for Thoracic Surgery; $M R$, mitral regurgitation; $M V$, mitral valve; $C A B G$, coronary artery bypass grafting; $C O R$, class of recommendation; $L O E$, levels of evidence; $I M R$, ischemic mitral valve regurgitation; $A V R$, aortic valve replacement; $L V$, left ventricle; $E D D$, end diastolic diameter; $N Y H A$, New York Heart Association; $G D M T$, goal-directed medical therapy.

chordal-sparing technique during the performance of mitral valve replacement as well as the use of an undersized, complete rigid annuloplasty ring for mitral valve repair in patients with IMR. The Guidelines for the performance of mitral valve replacement versus repair for IMR remain as follows:

- Mitral valve repair for IMR is performed with complete preservation of both anterior and posterior leaflet chords (COR I, LOE B).
- Mitral valve repair for IMR is performed with small, undersized, complete rigid annuloplasty ring (COR IIa, LOE B).

We are pleased to present these updated AATS Consensus Guidelines for Ischemic MR in light of the recently published follow-up results of the CTSN SMR and moderate MR clinical trials. We acknowledge that ischemic MR remains a challenging situation for clinicians and surgeons. However, emerging data have provided an opportunity for 
more guided recommendations for this patient population. As stated in our original Guidelines, the committee recommendations represent those based on data and expertise available at the current time. These Guidelines may continue to evolve as more data are reported in the future. Accordingly, we have submitted an update of our Guidelines for consideration to the AATS Council and the Journal of Thoracic and Cardiovascular Surgery.

\section{Conflict of Interest Statement}

D.H.A. has received institutional royalties for patents and institutional grants from Edwards Lifesciences, institutional royalties for patents and institutional grants from Medtronic, and institutional grants from NeoChord. G.A. reports personal fees from Abbott Vascular, Edwards Lifesciences, St Jude Medical, Mitralign, and Atricure. M.J.M. is coprincipal investigator of the Cardiovascular Outcomes Assessment of the MitralClip Percutaneous Therapy for Heart Failure Patients with Functional Mitral Regurgitation (COAPT) trial; Abbott Vascular is an uncompensated trial sponsor. All other authors have nothing to disclose with regard to commercial support.

\section{References}

1. American Association for Thoracic Surgery Ischemic Mitral Regurgitation Consensus Guidelines Writing C, Kron IL, Acker MA, Adams DH, Ailawadi G, Bolling SF, Hung JW, et al. 2015 The American Association for Thoracic Surgery Consensus Guidelines: ischemic mitral valve regurgitation. J Thorac Cardiovasc Surg. 2016;151:940-56.

2. Goldstein D, Moskowitz AJ, Gelijns AC, Ailawadi G, Parides MK, Perrault LP, et al. Two-year outcomes of surgical treatment of severe ischemic mitral regurgitation. N Engl J Med. 2016;374:344-53.

3. Michler RE, Smith PK, Parides MK, Ailawadi G, Thourani V, Moskowitz AJ, et al Two-year outcomes of surgical treatment of moderate ischemic mitral regurgitation. N Engl J Med. 2016;374:1932-41.

4. Kron IL, Hung J, Overbey JR, Bouchard D, Gelijns AC, Moskowitz AJ, et al. Predicting recurrent mitral regurgitation after mitral valve repair for severe ischemic mitral regurgitation. J Thorac Cardiovasc Surg. 2015;149: 752-61.e751.

5. Spoor MT, Geltz A, Bolling SF. Flexible versus nonflexible mitral valve rings for congestive heart failure: differential durability of repair. Circulation. 2006;114: I67-71.

6. Silberman S, Klutstein MW, Sabag T, Oren A, Fink D, Merin O, et al Repair of ischemic mitral regurgitation: comparison between flexible and rigid annuloplasty rings. Ann Thorac Surg. 2009;87:1721-6; discussion 1726-7.

7. Yun KL, Sintek CF, Miller DC, Schuyler GT, Fletcher AD, Pfeffer TA, et al. Randomized trial of partial versus complete chordal preservation methods of mitral valve replacement: a preliminary report. Circulation. 1999;100: II $90-4$. 